\title{
Publisher's Note: Interface coupling and growth rate measurements in multilayer Rayleigh-Taylor instabilities [Phys. Rev. Fluids 2, 062001(R) (2017)]
}

\author{
Raymond Adkins, Emily M. Shelton, Marie-Charlotte Renoult, Pierre Carles, \\ and Charles Rosenblatt \\ (Received 30 June 2017; published 17 July 2017)
}

DOI: 10.1103/PhysRevFluids.2.079901

This paper was published online on 19 June 2017 with a typographical error in the affiliation list. The second affiliation should read as "2 Laboratoire CORIA, Normandie Université, UNIROUEN, CNRS, LOMC, F-76000 Rouen, France.” The affiliation has been corrected online as of 5 July 2017. 